[Manuscript accepted for publication at Journal of Affective Disorders on 05/05/2021]

\title{
Optimal well-being in the aftermath of anxiety disorders:
}

\section{A 10-year longitudinal investigation}

David J. Disabato ${ }^{1}$, Todd B. Kashdan², James D. Doorley², Kerry C. Kelso ${ }^{2}$, Kristina M.

Volgenau $^{2}$, Andrew R. Devendorf ${ }^{3}$, and Jonathan Rottenberg ${ }^{3}$

Kent State University, Department of Psychological Sciences ${ }^{1}$

George Mason University, Department of Psychology ${ }^{2}$

University of South Florida, Department of Psychology ${ }^{3}$

\section{Corresponding Author:}

Todd B. Kashdan,

Department of Psychology, MS 3F5,

George Mason University,

Fairfax, VA 22030

tkashdan@gmu.edu 


\section{Introduction}

Anxiety disorders are common and disabling. The World Health Organization estimates that anxiety disorders interfere with daily function for 275 million people worldwide (Global Burden of Disease Collaborative Network, 2017). Two common anxiety disorders are Generalized Anxiety Disorder (GAD) and Panic Disorder (PD) with 12-month prevalence rates of $3.1 \%$ and $2.7 \%$, respectively (Kessler et al., 2005). GAD captures the chronic, less severe anxiety associated with worry and PD captures more brief and intense panic attacks along with the fear of future episodes and avoidance of activities to prevent them (Barlow, 2008). In this study, we use GAD and PD as exemplars of anxiety disorders.

Anxiety disorders are not necessarily chronic. In a nationally representative sample from the United States collected between 1990-1992, 29.4\% of people met criteria for anxiety disorders in the past 12 months. Only 30.5\% retained diagnostic criteria 11 years later (Kessler et al., 2005). Across 5 years, remission rates for anxiety disorders, such as generalized anxiety disorder (GAD), panic disorder (PD), and social anxiety disorder vary between 30 and 60\% (Yonkers, Dyck, Keller, 2001; Yonkers, Dyck, Warshaw, \& Keller, 2000). Because individuals fall below the diagnostic threshold over time, it can be presumed that some people not only recover from their anxiety disorder(s), but subsequently achieve good outcomes such as high levels of psychological well-being (optimal well being; OWB). Understanding good outcomes among anxiety disorders patients is crucial for understanding the mechanisms that are worthy targets of intervention. Before conducting more applied work, it is essential to address these fundamental gaps in knowledge. With this in mind, the present study estimated OWB prevalence rates following the diagnosis of two anxiety disorders (PD and GAD) in a nationally representative sample of the United States. 


\section{Optimal Well-being After Psychopathology}

Little is known regarding the prevalence of high levels of psychological well-being after a mental health diagnosis (Wood \& Tarrier, 2010; Rottenberg et al., 2018). Well-being can broadly be conceptualized as "[the] perceived enjoyment and fulfillment with one's life as a whole" (Disabato, Goodman, \& Kashdan, under review), and subsumes elements such as positive emotions (e.g., happiness), life satisfaction, purpose in life, and close social relationships (for different models of well-being, see Diener, 1984; Ryff, 1989; Keyes, Shmotkin, \& Ryff, 2002). Unfortunately, clinical psychology and psychiatry research has often not considered wellbeing as an endpoint (Wood \& Tarrier, 2010), which is part of a larger neglect of functional endpoints in outcome research (McKnight \& Kashdan, 2009).

By omitting well-being as an outcome, mental health fields lose important predictive and phenomenological information about psychopathology. Meta-analyses find that psychiatric symptoms correlate only modestly with functional impairment (McKnight \& Kashdan, 2009; Mcknight, Monfort, Kashdan, Blalock, \& Calton, 2016), suggesting that current symptomatic endpoints provide incomplete assessments of functioning. Studies also find that incorporating information about well-being into diagnostic endpoints can improve the prediction of mental health outcomes, above and beyond psychiatric symptoms alone (Keyes, Dhingra, \& Simoes, 2010; Rottenberg et al., 2019). Perhaps most important is that many patients with anxiety disorders have treatment goals that go beyond abatement of symptoms, like forming meaningful relationships, experiencing personal growth, and improving attitudes towards oneself (Holtforth, Wyss, Schulte, Trachsel, \& Michalak, 2009) -- all of which are components of well-being.

Given the importance of well-being as an outcome, our research team has drawn from models of well-being and human functioning (Diener, 1984; Ryan \& Deci, 2001; Ryff, 1989) to 
outline criteria to define OWB after psychology (Rottenberg et al., 2018). These criteria implement a population-based norms approach containing three elements: 1) a lifetime history of a mental health diagnosis, 2) the absence of a 12-month mental health diagnosis, and 3) high well-being, indicated by exceeding the top quartile of population-based norms on a battery of psychological-well-being measures (see Rottenberg et al., 2018 for extended discussion of the population-based approach).

\section{Anxiety vs. Depression}

Whether the prevalence rates of OWB differ across mental health diagnoses is not yet known. In general, data suggest that 50-70\% individuals with Major Depressive Disorder (MDD) recover within one year and only 6-15\% experience a chronic course (Richards, 2011). Using a nationally representative sample, our team found that $10 \%$ of individuals with a previous MDD diagnosis were not only symptom-free 10 years later, but also experienced OWB: well-being levels akin to the top 25\% of non-depressed adults in the United States (Rottenberg, Devendorf, Panaite, Disabato, \& Kashdan, 2019). We hypothesized that rates of OWB would be even higher for anxiety disorders for four reasons. First, in contrast to MDD, meta-analytic data suggest that distress related to anxiety disorders is only modestly correlated with the severity of functional impairment ( $r$ s $=.01$ to .46) (McKnight, Monfort, Kashdan, Blalock, Calton, 2016). Second, based on epidemiological data, MDD ranks as the $2^{\text {nd }}$ most burdensome disease in the U.S. in terms of years lived with disability (YLDs) whereas anxiety disorders rank $7^{\text {th }}$ (Vos et al., 2017). Third, the positive emotions, thoughts, and behaviors that are central to definitions of well-being are antithetical to the diagnostic features of MDD such as deficits in reward seeking and processing (i.e., anhedonia) (American Psychiatric Association, 2013). Meanwhile, reward deficits are not part of the diagnostic criteria for anxiety disorders. Finally, data suggest that 
depression is associated with greater negative stereotypes and mental health stigma than anxiety

(Wood, Birtel, Alsawy, Pyle, Morrison, 2014), pinpointing a cause of social rejection.

\section{Prediction of $\mathrm{OWB}$}

Moving from descriptive to predictive models, our goal was to predict the likelihood of OWB from information acquired at their intake assessment 10 years earlier (baseline). We tested a prediction model in people with GAD or PD that was previously supported for participants with MDD (Rottenberg, Devendorf, Panaite, Disabato, \& Kashdan, 2019): whether anxious individuals who experienced higher well-being at baseline would be more likely to be symptomfree and achieve optimal levels of psychological well-being 10 years later.

\section{Hypotheses}

We tested three hypotheses in the present study. First, we expected that OWB would be more common after anxiety disorders (GAD and PD) than MDD. Specifically, we predicted that the prevalence of OWB 10 years later among individuals with GAD or PD at baseline would be greater than $10 \%$, as this was the prevalence previously reported for MDD (Rottenberg, Devendorf, Panaite, Disabato, \& Kashdan, 2019). This hypothesis was pre-registered on September 24, 2018 (https://osf.io/puq8f/). Second, to test the relative prevalence of OWB among mentally healthy individuals, we tested the rudimentary hypothesis that people without GAD, PD, or MDD at baseline (i.e., healthy group) would have a higher prevalence of OWB 10

years later than those with one or more of those disorders at baseline (i.e., clinical group). Third, we tested whether baseline well-being predicted who reached OWB at follow-up among the individuals with an anxiety disorder (i.e., GAD and/or PD). The second and third hypotheses were not pre-registered and are considered exploratory.

\section{Methods}




\section{Participants and Procedures}

To test our hypothesis, Wave 1 and Wave 2 data were used from the Midlife Development in the United States study (MIDUS; http://midus.wisc.edu/scopeofstudy.php), a nationally representative sample of middle-aged (25-74 years) English-speaking adults recruited via a random-digit-dialing procedure (Brim, Ryff, \& Kessler, 2004). All MIDUS study procedures involving human participants were approved by Harvard Medical School in 1998 (IRB approval number: X112894-1). At Wave 1, all participants completed a 30-minute phone interview $(\mathrm{N}=3,487)$ and most completed a self-report survey $(\mathrm{n}=3,043)$. We focused on participants diagnosed with Generalized Anxiety Disorder (GAD), Panic Disorder (PD), or MDD at baseline $(\mathrm{N}=649)$ who completed all follow-up procedures 10 years later $(\mathrm{N}=256)$ : clinical group. For comparisons, we also used participants without GAD, PD, or MDD at baseline $(\mathrm{N}=$ 2838) who completed all follow-up procedures 10 years later $(\mathrm{N}=1207)$ : healthy group.

As expected for a 10-year follow-up, a large percentage of the MIDUS sample dropped out of the study and were missing data at Wave $2(58.0 \%)$. The rate of missing data did not differ across the clinical $(60.6 \%)$ and healthy $(57.5 \%)$ groups $(\chi 2=1.94, d f=1, p=.164)$. Table 1 contains the demographic information for those in the clinical group, healthy group, and those missing at Wave 2. The far right of the table also contains tests for significant differences between participants with data at follow-up (i.e., clinical and healthy group combined; $\mathrm{n}=1463$ ) vs. those missing at follow-up (i.e., missing data at Wave 2; $\mathrm{n}=2024$ ). All differences were significant: participants missing at follow-up were more likely to be younger, have less household income, be male, lack data on race/ethnicity, have less than a high school education, and be married. Note, the majority of missing/refused data for race at Wave 1 in the Missing at 
Wave 2 group is due to participant's completing the phone interview at Wave 1, but not completing the self-report survey at Wave 1 where race was assessed.

\section{Measures}

Diagnoses in the past 12 months were assessed during the phone interview at both waves using the Composite International Diagnostic Interview - Short Form (CIDI-SF), which was based on the SCID-III criteria. GAD was operationalized as worrying more than most people, most days, about more than one thing, and sometimes or often struggling to control that worry, for the past 12 months. PD was operationalized as two or more panic attacks in non-dangerous situations with several physiological symptoms over the past 12 months. Presence of MDD was operationalized as a two-week period in the past 12 months with depressed mood or anhedonia most of the day, nearly every day. Research demonstrates high inter-rater reliability for each CIDI-SF $(\mathrm{GAD}=.99, \mathrm{PD}=.98, \mathrm{MDD}=.93)($ Kessler, Andrews, Mroczek, Ustun, \& Wittchen, 1998). Symptom count scores for each CIDI-SF disorder were created and ranged from 0 to 10 for GAD, 0 to 6 for PD, and 0 to 7 for MDD. The Supplemental Materials contain details about the CIDI-SF interview questions and structure.

Well-being was measured via the self-report survey at both waves using items created by the MIDUS investigators and from the Scales of Psychological Well-being (SPWB; Ryff, 1989). All observed scores were created by unweighted averages of items, recoded such that higher scores reflect greater well-being. Life satisfaction items were rated on a 1-10 rating scale and included domain-specific health, work, children, and spouse/partner items as well as a single overarching rating. Affect items were rated on a 1-5 rating scale. Positive affect items included feeling cheerful, in good spirits, extremely happy, calm and peaceful, satisfied, and full of life. Negative affect items included feeling sad, nervous, restless/fidgety, hopeless, effortful, and 
worthless. The other six well-being dimensions were measured on a 1-7 rating scale by the SPWB: autonomy, environmental mastery, personal growth, positive relationships, purpose in life, and self-acceptance. The 3-item subscale version of the SPWB was used for Wave 1 and the 7-item subscale version for Wave 2. A general well-being total score was also created by converting the nine scales to Percentage of Maximum Possible (POMP) units and taking the unweighted average. The specific item wording and coefficient alphas of each scale are reported in the Supplemental Materials.

Consistent with emerging research, we operationalized OWB as 1) no GAD/PD/MDD symptoms at follow-up and 2) meeting objective well-being cutoff criteria using a populationbased approach (Rottenberg, Devendorf, Kashdan, \& Disabato, 2018; Rottenberg, Devendorf, Panaite, Disabato, \& Kashdan, 2019). The primary well-being cutoff criterion was consistent with prior research; it required 8 out of 9 well-being dimension scores at or above the $50^{\text {th }}$ percentile and 3 out of 9 scores at or above the $84^{\text {th }}$ percentile (i.e., the "strict" criterion). As sensitivity analyses to determine whether results were consistent with other well-being cutoffs, we included two less stringent well-being criteria: 8 out of 9 (i.e., the "medium" criterion) and 5 out of 9 (i.e., the "loose" criterion) well-being dimension scores at or above the $50^{\text {th }}$ percentile with neither requiring any scores at or above the $84^{\text {th }}$ percentile. Finally, we conducted a comprehensive examination of domain-specific OWB (i.e., scores at or above the $84^{\text {th }}$ percentile) across the nine well-being dimensions to determine which are the easiest for adults once diagnosed with GAD, PD, and MDD to experience 10 years later. These domain-specific tests of OWB are considered exploratory and presented in the Supplemental Materials.

\section{Data Analytic Plan}


To test the first hypothesis, proportions of OWB were calculated for each disorder: GAD, PD, and MDD. The primary test is for the strict well-being criterion; however, the medium and loose well-being criteria are provided as sensitivity analyses. Tests of differences between each pair of proportions (e.g., GAD vs. MDD) were conducted. Because many participants had diagnostic comorbidities, the groups were not independent. Therefore, a z-test that accounts for partially dependent groups was used (Derrick, Dobson-Mckittrick, Toher, \& White, 2015). For the second hypothesis, proportions of OWB were calculated for participants in the clinical group vs. healthy group. Because the groups were fully independent, traditional $\chi 2$-tests of the difference between proportions was used. Only participants with full data at both time points were included in analyses for tests of the first two hypotheses due to the difficulty with pooling results from statistical tests of differences in proportions with multiply imputed data.

Finally, the third hypothesis was tested with binary logistic regression models to determine whether general well-being at baseline predicted the probability of OWB 10 years later in participants with anxiety disorders (GAD and PD), after controlling for various demographic variables and baseline anxiety symptoms. To account for missing data from baseline to follow-up, multiple imputation by chained equations was used to estimate the regression coefficients, which decreases bias of parameter estimates and increases efficiency of standard errors (Buuren, 2018). One hundred imputed datasets were created via predictive mean matching and the results were pooled according to Rubin's rules (Rubin, 2011). In addition, repeated measures t-tests were conducted to confirm that OWB was associated with increases in well-being over time as opposed to simply starting with higher well-being at baseline.

\section{Results}

\section{Descriptive Statistics}


We first present comorbidity patterns in the clinical group. Table 2 presents the frequency counts and percentages of each disorder combination at baseline. The top two rows refer to participants with MDD and the bottom two rows refer to participants without MDD. The most common disorder combination was MDD only followed by PD only. The most common comorbidity was PD and MDD followed by GAD and MDD. The comorbidity of GAD and PD was the least common disorder combination, despite them both being anxiety disorders. The descriptive data for the baseline well-being POMP total score suggested a significant mean difference (Cohen's $d=1.06,(95 \% \mathrm{CI}=[0.90,1.23])$ between the clinical group $(M=0.66, S D$ $=0.14)$ and healthy group $(M=0.78 ; S D=0.10)$. Descriptive data for each of the 9 well-being scales is provided in the Supplemental Materials (i.e., Table S2). The largest differences were for the affect scales with the smallest differences for the autonomy, personal growth, and purpose in life scales.

\section{Hypothesis 1: OWB Comparing Anxiety vs. Depression}

We tested whether OWB after GAD or PD would be more common than OWB after MDD. Table 3 presents counts and percentages of OWB at 10-year follow-up across baseline disorders of GAD, PD, or MDD. Percentages with different letter superscripts are significantly different from each other at $\mathrm{p}<.05$. In general, OWB rates for PD were similar to MDD, while those for GAD were lower. The no symptom rates at follow-up are presented in the top rows of Table 3. Ten years later, $66.7 \%$ of participants with GAD, $60.2 \%$ with PD, and $63.6 \%$ with MDD no longer had symptoms of that particular disorder, suggesting relatively high singledisorder recovery rates. However, some participants retained or developed symptoms of one of the other two disorders, especially those diagnosed with GAD at baseline. While $45.9 \%$ of participants with PD and 51.3\% with MDD at baseline reported no symptoms of any disorder 10 
years later, the percentage was only $28.6 \%$ for GAD (GAD vs. PD: $d=-17.3 \%, z=-2.18, p=$ .029 ; GAD vs. MDD: $d=-22.7 \%, z=-3.04, p=.002)$.

The overall OWB rates that combined no symptoms at follow-up and the various wellbeing criteria are presented in the bottom rows of Table 3. Overall OWB rates declined with the increasingly stringent well-being criteria. Participants with GAD experienced the steepest decline for both the medium well-being criterion (GAD vs. PD: $d=-6.8 \%, z=-1.43, p=.152$; GAD vs. MDD: $d=-10.9 \%, z=-2.03, p=.043$ ) and strict well-being criterion (GAD vs. PD: $d=$ $-6.1 \%, z=-1.64, p=.101$; GAD vs. MDD: $d=-8.7 \%, z=-1.98, p=.047)$ PD and MDD had roughly similar rates of overall OWB based on each well-being criteria. When only comparing the participants who had no symptoms of any disorder 10 years later, those with GAD were not significantly less likely to meet the loose (GAD vs. PD: $d=-3.9 \%, \chi^{2}=-0.06, p=.806$; GAD vs. MDD: $d=-4.7 \%, z=-0.33, p=.738$ ), medium (GAD vs. PD: $d=-11.7 \%, \chi^{2}=-0.89, p=.345$; GAD vs. MDD: $d=-17.7 \%, z=-1.35, p=.176$ ) or strict (GAD vs. PD: $d=-13.3 \%, \chi 2=-1.79, p$ $=.181$; GAD vs. MDD: $d=-17.0 \%, z=-1.55, p=.121$ ) well-being criteria ${ }^{1}$.

\section{Hypothesis 2: OWB Comparing Clinical and Healthy Groups}

Table 3 also presents results for participants in the clinical versus healthy group. In general, OWB rates 10 years later for those in the clinical group were lower than the healthy group. The no symptoms of any disorder rate at follow-up was $52.3 \%$ for participants in the clinical group, while the rate was $84.6 \%$ for those in the healthy group $\left(d=-32.3 \%, \chi^{2}=132.13\right.$, $p<.001)$. Overall OWB rates were lower among those in the clinical group for the loose $(d=-$ $\left.25.1 \%, \chi^{2}=53.18, p<.001\right)$, medium $\left(d=-17.9 \%, \chi^{2}=34.14, p<.001\right)$ and $\operatorname{strict}(d=-11.4 \%$,

\footnotetext{
${ }^{1}$ Due to only one GAD and PD comorbidity within the no symptoms 10 years later subsample, traditional tests for difference in proportions were used as the test that accounts for subsample dependencies (i.e., comorbidity) requires at least 2 paired cases.
} 
$\chi 2=19.00, p<.001)$ well-being criteria. When only comparing the participants who had no symptoms 10 years later, those in the clinical group were less likely to meet the loose $(d=-6.3 \%$, $\left.\chi^{2}=2.11, p=.146\right)$, medium $\left(d=-12.1 \%, \chi^{2}=7.63, p=.006\right)$ and $\operatorname{strict}(d=-7.5 \%, \chi 2=3.88, p$ $=.049)$ well-being criteria.

\section{Hypothesis 3: Prediction of $\mathrm{OWB}$}

We next tested whether baseline well-being predicted the likelihood of overall OWB for participants with GAD or PD. The complete data cases were participants in the clinical group with an anxiety disorder $(\mathrm{n}=124)$, although multiple imputation was used to incorporate participants missing at Wave 2 who had GAD or PD at baseline. Overall OWB (based on the three different well-being criteria as well as no symptoms of any disorder) was the outcome, the general well-being POMP total score at baseline was the focal predictor, and demographics (i.e., sex, age, education), mental health treatment the year leading up to Wave $2^{2}$, and anxiety symptoms were covariates. Sex and GAD symptoms had to be removed from the strict wellbeing criterion regression because there were no males or people with GAD who met the OWB criteria resulting in unstable coefficients. Results in Table 4 indicate that baseline well-being marginally predicted the primary strict well-being criterion; however, the association was nonsignificant for the alternative medium or loose well-being criteria as well as no symptoms of any disorder at follow-up. While both GAD and PD symptoms significantly predicted no symptoms at follow-up, they did not for any of the well-being criteria. For those 1 SD below and above the mean on baseline well-being, their fitted probabilities of OWB were $0.3 \%$ vs. $4.1 \%$ for the strict, $1.5 \%$ vs. $5.4 \%$ for the medium, and $16.3 \%$ vs. $24.2 \%$ for the loose well-being criteria, while it

\footnotetext{
${ }^{2}$ Mental health treatment was quantified as a count variable of the number of times the participant saw a professional for an emotional or psychiatric problem in the past 12 months (Median $=1 ; \mathrm{IQR}=[0,4]$, Range $=[0$, 122]). Professionals included psychiatrists, general practitioners or other medical doctors, psychologists/counselors/therapists, or spiritual healers (e.g., minister, rabbi).
} 
was $38.8 \%$ vs. $39.7 \%$ for no symptoms of any disorder. Well-being was a significant predictor of OWB when only the three demographics were included as covariates: strict $(b=8.39, s e=4.20$, $p=.050)$, medium $(b=5.44, s e=2.71 ; p=.047)$, or loose $(b=2.92, s e=1.42 ; p=.042)$ wellbeing criteria as well as no symptoms of any disorder $(b=2.70, s e=1.12, p=.017)$.

Finally, we tested whether participants with GAD or PD who we classified as reaching OWB reported increases in well-being over 10 years rather than simply starting higher on wellbeing at baseline. Table 5 reports the means, standard deviations, and Cohen's d values for the well-being POMP total score across each Wave and classification of participants as either a) having symptoms of any disorder at Wave 2, b) having no symptoms of any disorder, but not meeting the well-being criteria, or c) also meeting the well-being criteria. Those with OWB reported large increases in well-being from Wave 1 to Wave 2, In contrast, there were only small increases in well-being for participants with any symptoms at follow-up $(n=68)$ : Cohen's $d=$ $.16(p=.201)$ from Wave $1(M=0.60, S D=0.15)$ to Wave $2(M=0.62, S D=0.15)$. Mixed factorial ANOVAs tested whether there was differential change over time across the participant classifications. The Wave * classification interaction was non-significant for the strict well-being criterion $(F(2,121)=2.65, p=.075)$ but significant for the medium $(F(2,121)=3.65, p=.039)$ and loose $(F(2,121)=10.04, p<.001)$ well-being criteria.

\section{Discussion}

We tested three hypotheses related to optimal well-being (OWB) after anxiety disorders (GAD or PD) in a nationally representative sample from the United States. Our first, preregistered hypothesis that OWB at follow-up 10 years later was more likely after anxiety than depression was not supported. The primary test was for the strict well-being criterion; however, the medium and loose well-being criteria were provided as sensitivity analyses. We observed 
results opposite to our hypothesis, regardless of the well-being criteria. A greater percentage of adults experienced OWB 10 years after MDD relative to GAD. Adults recovering from PD exhibited OWB rates lower than MDD but not as low as GAD. Our second hypothesis that OWB was more likely for participants without any disorders at baseline (healthy group) compared to those with a disorder(s) at baseline (clinical group) was supported. Overall OWB rates in the healthy group were double or more those in the clinical group. Our third hypothesis that baseline well-being, while meeting diagnostic criteria for GAD or PD, would predict OWB 10 years later was not supported. Baseline well-being did not significantly predict OWB at follow-up after controlling for anxiety symptoms and mental health treatment. Overall, the patterns of findings were similar regardless of how stringent the well-being cutoffs were.

\section{Overall OWB}

The initial hurdle of reaching OWB was having no symptoms at follow-up. Over $60 \%$ of participants reported no symptoms of their baseline disorder a decade later, which is consistent with prior research demonstrating the majority of people with MDD, PD' and GAD no longer meet diagnostic criteria for that particular disorder in the long term (Fuller-Thomson, Battiston, Gadalla, \& Brennenstuhl, 2014; Andersch \& Hetta, 2002; Yonkers, Bruce, Dyck, Keller, 2003). While the present findings demonstrate comparable rates of recovery between disorders, diagnostic differences emerged when examining participants who did not report symptoms of any of the three disorders. Relative to MDD and PD, disproportionately fewer people were asymptomatic of all disorders after GAD, meaning symptoms of MDD and/or PD persisted or emerged even though someone had no symptoms of GAD. This is consistent with newer conceptual models of psychopathology which suggest manifestations of psychopathology (e.g., specific disorder criteria) can change over time, even if underlying transdiagnostic features of 
psychopathology (e.g., frequent negative emotions, negative repetitive thinking, experiential avoidance, etc.) remain constant (Krueger \& Eaton, 2015).

To qualify as reaching OWB, participants needed to report elevated well-being in addition to being asymptomatic of all disorders. Regardless of which well-being criteria was used, fewer participants with GAD at baseline had OWB compared to those with MDD. Under the strict well-being criterion, $0 \%$ participants had OWB after GAD. In contrast, $9 \%$ had OWB after MDD, which is comparable to our previous result ${ }^{3}$ (10\%; Rottenberg, Devendorf, Panaite, Disabato, \& Kashdan, 2019). Our results suggest that the differences in overall OWB rates between GAD and MDD may be solely due to differences in no symptom of any disorder rates. More research is needed to clarify the degree to which no symptoms vs. well-being deficits explain the overall OWB differences across anxiety and depressive disorders.

\section{GAD vs. MDD}

After our pre-registered hypothesis was not supported, we considered empirical and theoretical reasons to suggest rates of OWB would indeed be higher after depression than anxiety. In individuals with comorbid anxiety and depression, GAD has a uniquely detrimental impact on well-being (Mittal, Fortney, Pyne, Edlund, \& Wetherell, 2006), which persists after depressive symptoms remit (Misri \& Swift, 2015). Even if someone is currently asymptomatic, a former diagnosis of GAD may continue to undermine their ability to thrive, indicating a particular need for interventions designed to foster well-being. Likewise, existing treatments could incorporate well-being interventions alongside those aimed at reducing symptoms to improve the trajectories of people with GAD.

\footnotetext{
${ }^{3}$ Even though Rottenberg et al. (2019) and the present study used the same MIDUS dataset, the percentage is slightly smaller in the present study because OWB after MDD required no symptoms of MDD, PD, or GAD in the present study while OWB after MDD required only no symptoms of MDD in Rottenberg et al. (2019).
} 
Subtle cognitive and affective differences between MDD and GAD may explain why OWB is less common for people after GAD. Repetitive negative thinking is elevated in both disorders, yet people with MDD report higher levels of rumination, or negative thoughts about the past, while people with GAD report higher levels of worry, or negative thoughts about future threats (Hendriks et al., 2014). Worry may be more detrimental to recovery and well-being than rumination. The rumination from dwelling on past negative life events in MDD may dissipate as the time since the life event (e.g., divorce) grows longer, opening the door for future well-being. This could be particularly true for the $\sim 50 \%$ of people with MDD who never experience a second depressive episode and may be characterized better as struggling with adjustment as opposed to true psychopathology (Lorenzo-Luaces, 2015). Surprisingly, even previously meeting the criterion for the suicidal ideation MDD symptom may not prevent elevated well-being later on if the suicidal ideation has been gone for at least a year (Bryan, Bryan, \& Kopacz, 2021). In contrast, the process of new, evolving worries in GAD may reflect enduring personality patterns (Mahaffey, Watson, Clark, \& Kotov, 2016). People with GAD also tend to be more reactive to their experiences than people with MDD. GAD symptoms uniquely predict emotional reactivity to present-moment thoughts while MDD symptoms uniquely predict a lack of present-moment awareness (Curtiss \& Klemanski, 2014). Similarly, people with GAD demonstrate greater emotional reactivity to negative stimuli and worst imagined outcomes compared to those with MDD, who display blunted emotional reactivity (Macnamara, Kotov, \& Hajcak, 2015). Perhaps increased emotional reactivity in GAD - in the form of worry - compared to blunted reactivity in MDD is more pernicious, reducing well-being in the long term.

The simplest explanation stems from the diagnostic criteria themselves. MDD is an episodic disorder characterized by periods of low or no symptoms punctuated by full-fledged 
pathology. Diagnostic criteria for a single episode require symptoms to be present most-of-theday nearly every day for only two weeks (American Psychiatric Association, 2013). In contrast, GAD is not episodic and requires symptoms more days than not anywhere from 6-12 months. People who report GAD symptoms lasting six months or more compared to three months display a more severe and chronic course of illness (Burstein, Beesdo-Baum, He, \& Merikangas, 2014). Diagnostic criteria for GAD may capture inherently more enduring psychopathology by requiring symptoms to last many times longer than MDD. It is unclear whether more chronic courses of clinical depression (e.g., Persistent Depressive Disorder) would experience similar overall OWB rates as GAD.

\section{Clinical versus healthy group}

Given the sometimes chronic and fluctuating course of psychopathology (Olino, Klein, Lewinsohn, Rohde, \& Seeley, 2010), it is not surprising that OWB 10 years later was higher in the healthy compared to the clinical group. Even among those with no symptoms of any disorder at follow-up, those in the clinical group were significantly less likely to reach the strict wellbeing criterion. The present finding reiterates that full symptom recovery does not guarantee high well-being, and that people with priori psychopathology do not automatically experience psychological well-being after symptoms have remitted. Mental health treatments aimed solely at symptom reduction may benefit from the inclusion of interventions designed to help people thrive. Few people in the clinical group met the criteria for OWB in the domains of negative and positive affect. Affect may be the most difficult dimensions of well-being for individuals with psychopathology to reach very high levels of. This is consistent with emotional dysfunction being at the core of anxiety and depression (Coifman \& Summers, 2019). However, people with psychopathology value other aspects of their well-being besides affect. Patients with depression 
report wanting more positive self-perceptions (e.g., self-acceptance), healthy social functioning (e.g., positive relationships), and agency (e.g., autonomy) (Chevance et al., 2020). These nonaffective dimensions of well-being may be more common for people with prior psychopathology to reach high levels of. The baseline mean differences in well-being between the clinical group and healthy group indicate the smallest differences for autonomy, personal growth, and purpose in life. Interventions that target these well-being dimensions may be fruitful for helping people live rich and meaningful lives after psychopathology (e.g., well-being therapy; Fava, 2016).

\section{Who experiences $\mathrm{OWB}$ ?}

We found that baseline well-being did not predict a greater likelihood of OWB 10 years later. While the impact of baseline well-being was significant when only controlling for demographics, the impact decreased and become non-significant upon controlling for baseline anxiety symptom severity. Surprisingly, anxiety symptoms were not significant either suggesting that perhaps OWB after anxiety is caused by phenomena not measured in the present study. This contrasts with what was found for depression where baseline well-being retained a significant effect on OWB after accounting for both baseline depressive and anxiety symptoms (Rottenberg, Devendorf, Panaite, Disabato, \& Kashdan, 2019). Note, the discrepancy is not solely due to the complete cases sample sizes $(n=124$ vs. $n=239)$ since similar predictors resulted in only half the Pseudo- $\mathrm{R}^{2}$ for anxiety (18.5\%) compared with depression (40.7\%). Although transdiagnostic models are popular within research on internalizing psychopathology, this discrepancy in findings highlights that the path to OWB may differ across anxiety and depressive disorders. While the path from experiencing MDD to well-being may involve a return to reacting to emotionally provocative stimuli, the path from anxiety disorders to well-being may involve a reduction in emotional reactivity. Regardless of how someone reaches OWB, we found that 
participants with OWB after anxiety experienced significant increases in well-being over time and did not simply start with higher well-being at baseline.

\section{Limitations and Future Directions}

Several limitations should be noted. First, the MIDUS study used the CIDI-SF to assign psychiatric diagnoses. The assessment criteria do not align perfectly with the DSM-III-R criteria (or the DSM-5) (American Psychiatric Association, 2013). For example, CIDI-SF items fail to assess persistent worry about having another panic attack, concerns about the implications of panic symptoms (e.g., "I’m going crazy", "I'm having a heart attack”), or behavioral changes due to panic attacks (e.g., excessive avoidance). As such, results related to PD should be interpreted with caution. When applying stricter, DSM criteria, the prevalence of OWB may be lower than found here for PD. Second, attrition from Wave 1 to 2 led to relatively small subsamples of individuals with full data at Wave 2 who met diagnostic criteria for anxiety or depressive disorders at Wave 1. For this reason, as well as OWB after psychiatric diagnoses being a new area of research, we elected to minimize Type II errors and not to control for multiple comparisons; however, Type I errors are still a concern and future researchers will need to replicate our findings - especially those involving domain-specific OWB. Nevertheless, it is possible that participants who were most burdened by mental health concerns were too impaired to complete Wave 2 measures, resulting in biased rates of remission and OWB. Differential OWB rates across GAD, PD, MDD and other disorders warrant further investigation with larger samples and multi-method measurement approaches of high functioning/well-being (e.g., informant reports, experience sampling in daily life; Rottenberg, Devendorf, Kashdan, \& Disabato, 2018). We hope this work inspires greater interest in the possibility of high functioning after psychopathology. Researchers and clinicians must seek to better understand contributors to 
OWB, which may be less common in GAD, to eventually increase the well-being of those diagnosed with anxiety and depressive disorders in the United States. 


\section{References}

American Psychiatric Association (2013). Diagnostic and statistical manual of mental disorders (DSM-5). Washington, DC: American Psychiatric Publishing.

Andersch, S., Hetta, J. (2002). A naturalistic fifteen-year follow-up study of panic disorder patients. European Psychiatry, 17(S1), 166s.

Barlow, D. H. (2008). Clinical handbook of psychological disorders: A step-by-step treatment manual. New York, NY: Guildford Press.

Brim, O. G., Ryff, C. D., \& Kessler, R. C. (2004). The MIDUS National Survey: An Overview. In O. G. Brim, C. D. Ryff, \& R. C. Kessler (Eds.), The John D. and Catherine T. MacArthur foundation series on mental health and development. Studies on successful midlife development. How healthy are we? A national study of well-being at midlife (p. 1-34). The University of Chicago Press.

Bryan, C. J., Bryan, A. O., \& Kopacz, M. S. (2021). Finding purpose and happiness after recovery from suicide ideation. The Journal of Positive Psychology, 16(1), 46-53.

Burstein, M., Beesdo-Baum, K., He, J-P, \& Merikangas, K. R. (2014). Threshold and subthreshold generalized anxiety disorder among US adolescents: Prevalence, sociodemographic, and clinical characteristics. Psychological Medicine, 44(11), 23512362.

Buuren, S. V. (2018). Flexible Imputation of Missing Data (Second Edition). London, UK: CRC Press.

Chevance, A., Ravaud, P., Tomlinson, A., Le Berre, C., Teufer, B., Touboul, S., ... \& Tran, V. T. (2020). Identifying outcomes for depression that matter to patients, informal caregivers, 
and health-care professionals: qualitative content analysis of a large international online survey. The Lancet Psychiatry, 7(8), 692-702.

Coifman, K. G., \& Summers, C. B. (2019). Understanding emotion inflexibility in risk for affective disease: Integrating current research and finding a path forward. Frontiers in Psychology, 10, 392.

Curtiss, J., Klemanski, D. H. (2014). Teasing apart low mindfulness: Differentiating deficits in mindfulness and in psychological flexibility in predicting symptoms of generalized anxiety disorder and depression. Journal of Affective Disorders, 166, 41-47.

Derrick, B., Dobson-Mckittrick, A., Toher, D., \& White, P. (2015) Test statistics for comparing two proportions with partially overlapping samples. Journal of Applied Quantitative Methods, 10(3), 1-14.

Diener, E. (1984). Subjective Well-Being. Psychological Bulletin, 95(3), 542-575.

Disabato, D. J., Goodman, F. R., \& Kashdan, T. B. (under review). A hierarchical framework for the measurement of well-being. Personality and Social Psychology Review.

Fava, G. A. (2016). Well-being therapy: Treatment manual and clinical applications. New York, NY: Karger.

Fuller-Thomson, E., Battiston, M., Gadalla, T. M., \& Brennenstuhl, S. (2014) Bouncing back: Remission from depression in a 12-year panel study of a representative Canadian community sample. Social Psychiatry and Psychiatric Epidemiology, 49(6), 903-910.

Global Burden of Disease Collaborative Network (2017). Global Burden of Disease Study 2016: Incidence, Prevalence, and Years Lived with Disability 1990-2016. Institute for Health Metrics and Evaluation (IHME); Seattle, United States. 
Hendriks, S. M., Licht, C. M., Spijker, J., Beekman, A. T., Hardeveld, F., de Graaf, R., \& Penninx, B. W. (2014). Disorder-specific cognitive profiles in major depressive disorder and generalized anxiety disorder. BMC Psychiatry, 14(1), 1-9.

Hoge, E. A., Bui, E., Marques, L., Metcalf, C. A., Morris, L. K., Robinaugh, D. J., ... \& Simon, N. M. (2013). Randomized controlled trial of mindfulness meditation for generalized anxiety disorder: Effects on anxiety and stress reactivity. The Journal of Clinical Psychiatry, 74(8), 786-792.

Holtforth, M. G., Wyss, T., Schulte, D., Trachsel, M., \& Michalak, J. (2009). Some like it specific: The difference between treatment goals of anxious and depressed patients. Psychology and Psychotherapy: Theory, Research and Practice, 82(3), 279-290.

Kessler, R. C., Andrews, G., Mroczek, D., Ustun, B., Wittchen, H-U (1998). The World Health Organization Composite International Diagnostic Interview short-form (CIDI-SF). International Journal of Methods in Psychiatric Research, 7(4), 171-185.

Kessler, R. C., Chiu, W. T., Demler, O., \& Walters, E. E. (2005). Prevalence, severity, and comorbidity of 12-month DSM-IV disorders in the National Comorbidity Survey Replication. Archives of General Psychiatry, 62(6), 617-627.

Keyes, C. L., Dhingra, S. S., \& Simoes, E. J. (2010). Change in level of positive mental health as a predictor of future risk of mental illness. American Journal of Public Health, 100(12), 2366-2371.

Keyes, C. L., Shmotkin, D., \& Ryff, C. D. (2002). Optimizing well-being: the empirical encounter of two traditions. Journal of Personality and Social Psychology, 82(6), 10071022. 
Krueger, R. F., \& Eaton, N. R. (2015). Transdiagnostic factors of mental disorders. World Psychiatry, 14(1), 27-29.

Lorezno-Luaces, L. (2015). Heterogeneity in the prognosis of major depression: From the common cold to a highly debilitating and recurrent illness. Epidemiology and Psychiatric Sciences, 24, 466-472.

Macnamara, A., Kotov, R., Hajcak, G. (2015). Diagnostic and Symptom-Based Predictors of Emotional Processing in Generalized Anxiety Disorder and Major Depressive Disorder: An Event-Related Potential Study. Cognitive Therapy and Research, 40(3), 275-289.

Mahaffey, B. L., Watson, D., Clark, L. A., \& Kotov, R. (2016). Clinical and personality traits in emotional disorders: Evidence of a common framework. Journal of Abnormal Psychology, 125(6), 758-767.

McKnight, P. E., \& Kashdan, T. B. (2009). The importance of functional impairment to mental health outcomes: a case for reassessing our goals in depression treatment research. Clinical Psychology Review, 29(3), 243-259.

Mcknight, P. E., Monfort, S. S., Kashdan, T. B., Blalock, D. V., \& Calton, J. M. (2016) Anxiety symptoms and functional impairment: A systematic review of the correlation between the two measures. Clinical Psychology Review, 45, 115-130.

Misri, S., Swift, E. (2015). Generalized Anxiety Disorder and Major Depressive Disorder in Pregnant and Postpartum Women: Maternal Quality of Life and Treatment Outcomes. Journal of Obstetrics and Gynaecology Canada, 37(9), 798-803.

Mittal, D., Fortney, J. C., Pyne, J. M., Edlund, M. J., \& Wetherell, J. L. (2006). Impact of Comorbid Anxiety Disorders on Health-Related Quality of Life Among Patients With Major Depressive Disorder. Psychiatric Services, 57(12), 1731-1737. 
Olino, T. M., Klein, D. N., Lewinsohn, P. M., Rohde, P., \& Seeley, J. R. (2010). Latent trajectory classes of depressive and anxiety disorders from adolescence to adulthood: descriptions of classes and associations with risk factors. Comprehensive Psychiatry, 51(3), 224-235.

Richards, D. (2011). Prevalence and clinical course of depression: A review. Clinical Psychology Review, 31(7), 1117-1125.

Rottenberg, J., Devendorf, A. R., Kashdan, T. B., Disabato, D. J. (2018). The Curious Neglect of High Functioning After Psychopathology: The Case of Depression. Perspectives on Psychological Science, 13(5), 549-566.

Rottenberg, J., Devendorf, A. R., Panaite, V., Disabato, D. J., Kashdan, T. B. (2019). Optimal Well-Being After Major Depression. Clinical Psychological Science, 7(3), 621-627.

Rubin, D. B. (2011). Multiple Imputation for Nonresponse in Surveys. Hoboken, NJ: John Wiley. Ryan, R. M., \& Deci, E. L. (2001). On happiness and human potentials: A review of research on hedonic and eudaimonic well-being. Annual Review of Psychology, 52(1), 141-166.

Ryff, C. D. (1989). Happiness is everything, or is it? Explorations on the meaning of psychological well-being. Journal of Personality and Social Psychology, 57(6), 10691081.

Vos, T., Abajobir, A. A., Abate, K. H., Abbafati, C., Abbas, K. M., Abd-Allah, F., ... \& Aboyans, V. (2017). Global, regional, and national incidence, prevalence, and years lived with disability for 328 diseases and injuries for 195 countries, 1990-2016: a systematic analysis for the Global Burden of Disease Study 2016. The Lancet, 390(10100), 12111259. 
Wood, L., Birtel, M., Alsawy, S., Pyle, M., Morrison, A. (2014). Public perceptions of stigma towards people with schizophrenia, depression, and anxiety. Psychiatry Research, 220(12), 604-608.

Wood, A. M., \& Tarrier, N. (2010). Positive clinical psychology: A new vision and strategy for integrated research and practice. Clinical Psychology Review, 30(7), 819-829.

Yonkers K. A., Dyck, I. R., Warshaw, M., \& Keller, M. B. (2000). Factors predicting the clinical course of generalised anxiety disorder. British Journal of Psychiatry, 176(6), 544-549.

Yonkers, K. A., Bruce, S. E., Dyck, I. R., \& Keller, M. B. (2003). Chronicity, relapse, and illness? Course of panic disorder, social phobia, and generalized anxiety disorder: Findings in men and women from 8 years of follow-up. Depression and Anxiety, 17(3), $173-179$.

Yonkers, K. A., Dyck, I. R., \& Keller, M. B. (2001). An Eight-Year Longitudinal Comparison of Clinical Course and Characteristics of Social Phobia Among Men and Women. Psychiatric Services, 52(5), 637-643. 


\section{Tables}

Table 1. Sample demographics across study groups.

\begin{tabular}{|c|c|c|c|c|c|c|c|}
\hline & \multicolumn{2}{|c|}{$\begin{array}{l}\text { Clinical Group } \\
\quad(N=256)\end{array}$} & \multicolumn{2}{|c|}{$\begin{array}{l}\text { Healthy Group } \\
(\mathrm{N}=1207)\end{array}$} & \multicolumn{2}{|c|}{$\begin{array}{c}\text { Missing at Wave } 2 \\
(\mathrm{~N}=\mathbf{2 0 2 4})\end{array}$} & $\begin{array}{l}\text { Observed vs. } \\
\text { Missing }\end{array}$ \\
\hline \multirow[t]{2}{*}{$\begin{array}{l}\text { Age (median \& IQR) } \\
\text { Income (median \& IQR) }\end{array}$} & \multicolumn{2}{|c|}{$\begin{array}{l}44[36,52] \\
45 \mathrm{k}[26 \mathrm{k}, 87 \mathrm{k}]\end{array}$} & \multicolumn{2}{|c|}{$\begin{array}{l}47[37,57] \\
60 \mathrm{k}[33 \mathrm{k}, 98 \mathrm{k}] \\
\end{array}$} & \multicolumn{2}{|c|}{$\begin{array}{l}45[34,57] \\
43 \mathrm{k}[23 \mathrm{k}, 78 \mathrm{k}]\end{array}$} & $\begin{array}{l}\chi 2(1)=4.76^{*} \\
\chi 2(1)=37.65^{* * *}\end{array}$ \\
\hline & Number & Percent & Number & Percent & Numbe & $\underline{\text { Percent }}$ & \\
\hline Sex & - & & - & & - & & $\chi 2(1)=7.00^{* *}$ \\
\hline Female & 177 & $69.1 \%$ & 603 & $50.0 \%$ & 986 & $48.7 \%$ & \\
\hline Male & 79 & $30.9 \%$ & 604 & $50.0 \%$ & 1038 & $51.3 \%$ & \\
\hline Race & & & & & & & $\chi 2(5)=67.27^{* * *}$ \\
\hline White & 233 & $91.0 \%$ & 1110 & $92.0 \%$ & 1243 & $61.4 \%$ & \\
\hline African American & 6 & $2.3 \%$ & 50 & $4.1 \%$ & 145 & $7.2 \%$ & \\
\hline Native American & 2 & $0.8 \%$ & 7 & $0.6 \%$ & 13 & $0.6 \%$ & \\
\hline Asian/Pacific Islander & 1 & $0.4 \%$ & 9 & $0.7 \%$ & 24 & $0.1 \%$ & \\
\hline Other & 9 & $3.5 \%$ & 13 & $1.1 \%$ & 58 & $0.3 \%$ & \\
\hline Multiracial & 1 & $0.4 \%$ & 7 & $0.6 \%$ & 15 & $0.7 \%$ & \\
\hline Missing/Refused & 4 & $1.6 \%$ & 11 & $0.9 \%$ & 526 & $26.0 \%$ & \\
\hline Education & & & & & & & $\chi 2(3)=120.37 * * *$ \\
\hline Less than high school & 20 & $7.8 \%$ & 63 & $5.2 \%$ & 297 & $14.7 \%$ & \\
\hline Graduated high school & 72 & $28.1 \%$ & 456 & $37.8 \%$ & 598 & $29.5 \%$ & \\
\hline Some college & 90 & $35.2 \%$ & 338 & $28.0 \%$ & 665 & $32.9 \%$ & \\
\hline Graduated college & 74 & $28.9 \%$ & 350 & $29.0 \%$ & 462 & $22.8 \%$ & \\
\hline Marital Status & & & & & & & $\chi 2(4)=61.00^{* * *}$ \\
\hline Married & 135 & $52.7 \%$ & 867 & $71.8 \%$ & 1002 & $68.5 \%$ & \\
\hline Separated & 13 & $5.1 \%$ & 13 & $1.1 \%$ & 26 & $1.8 \%$ & \\
\hline Divorced & 68 & $26.6 \%$ & 160 & $13.3 \%$ & 228 & $15.6 \%$ & \\
\hline Widowed & 11 & $4.3 \%$ & 50 & $4.1 \%$ & 61 & $4.2 \%$ & \\
\hline Never Married & 29 & $11.3 \%$ & 117 & $9.7 \%$ & 146 & $10.0 \%$ & \\
\hline
\end{tabular}

Note. The "Observed vs. Missing" column presents significant tests. The age and income tests are Mood's test of median differences and sex, race, education, and marital status tests are chi-square tests of independence. 
Table 2. Diagnostic comorbidity in the clinical group at Wave 1.

\begin{tabular}{l|ll}
\hline & \multicolumn{2}{|c}{ Clinical Group } \\
\hline MDD No & No PD & Yes PD \\
\hline No GAD & $0(0.0 \%)$ & $46(18.0 \%)$ \\
Yes GAD & $10(3.9 \%)$ & $5(2.0 \%)$ \\
\hline MDD Yes & No PD & Yes PD \\
\hline No GAD & $132(51.6 \%)$ & $36(14.1 \%)$ \\
Yes GAD & $16(6.3 \%)$ & $11(4.3 \%)$ \\
\hline
\end{tabular}

TOTAL Dx: $\mathrm{MDD}=195, \mathrm{PD}=98, \mathrm{GAD}=42$

Note. GAD $=$ Generalized Anxiety Disorder; $\mathrm{PD}=$ Panic Disorder; MDD = Major Depressive Disorder; Percentages of of the total frequency; $\mathrm{Dx}=$ disorders. 
Table 3. Optimal well-being (OWB) rates across disorders at Wave 2.

\begin{tabular}{|c|c|c|c|c|c|c|c|c|c|c|}
\hline \multirow[b]{2}{*}{ OWB OPERATION } & \multicolumn{2}{|c|}{ GAD } & \multicolumn{2}{|c|}{ PD } & \multicolumn{2}{|c|}{ MDD } & \multicolumn{2}{|c|}{ Clinical group } & \multicolumn{2}{|c|}{ Healthy group } \\
\hline & Count & Percent & Count & Percent & Count & Percent & Count & Percent & Count & Percent \\
\hline Data at wave 1 and 2 & 42 & $100.0 \%$ & 98 & $100.0 \%$ & 195 & $100.0 \%$ & 256 & $100.0 \%$ & 1207 & $100.0 \%$ \\
\hline No past disorder symptoms & 28 & $66.7 \%$ & 59 & $60.2 \%$ & 124 & $63.6 \%$ & NA & NA & NA & NA \\
\hline No GAD/PD/MDD symptoms & 12 & $28.6 \% \mathrm{o}^{\mathrm{a}}$ & 45 & $45.9 \% \mathrm{o}^{\mathrm{b}}$ & 100 & $51.3 \%{ }^{\mathrm{b}}$ & 134 & $52.3 \% \mathrm{o}^{\mathrm{a}}$ & 1021 & $84.6 \%{ }^{\mathrm{b}}$ \\
\hline Loose well-being criterion & 7 & $16.7 \% \%^{\mathrm{a}}$ & 28 & $28.6 \%{ }^{\mathrm{ab}}$ & 63 & $32.3 \% \%^{\mathrm{b}}$ & 82 & $32.0 \% 0^{\mathrm{a}}$ & 689 & $57.1 \%{ }^{\mathrm{b}}$ \\
\hline Medium well-being criterion & 1 & $2.4 \%{ }^{\mathrm{a}}$ & 9 & $9.2 \%{ }^{\mathrm{ab}}$ & 26 & $13.3 \%{ }^{\mathrm{b}}$ & 32 & $12.5 \%^{\mathrm{a}}$ & 367 & $30.4 \%{ }^{b}$ \\
\hline Strict well-being criterion & 0 & $0.0 \%^{\mathrm{a}}$ & 6 & $6.1 \%{ }^{\mathrm{ab}}$ & 17 & $8.7 \%^{\mathrm{b}}$ & 21 & $8.2 \%^{\mathrm{a}}$ & 237 & $19.6 \% \mathrm{~b}$ \\
\hline
\end{tabular}

Note. GAD = generalized anxiety disorder; $\mathrm{PD}=$ panic disorder; $\mathrm{MDD}=$ major depressive disorder; Clinical group $=\mathrm{GAD}$ and/or PD and/or MDD; Healthy group = No GAD and no PD and no MDD; ${ }^{\text {a,b }}$ differently lettered superscripts indicate significantly different proportions $(\mathrm{p}<.05)$ and are separate for 1$)$ GAD vs. PD vs. MDD and 2) Clinical group vs. Healthy group. 
Table 4. Logistic regression results of well-being at Wave 1 predicting optimal well-being (OWB) at Wave 2.

\begin{tabular}{|c|c|c|c|c|c|c|c|c|c|c|c|c|}
\hline \multirow[b]{2}{*}{ Predictor } & \multicolumn{6}{|c|}{ Strict well-being criterion $\left(\right.$ Pseudo- $\mathbf{R}^{2}=.185$ ) } & \multicolumn{6}{|c|}{ Medium well-being criterion $\left(\right.$ Pseudo- $\left.R^{2}=.176\right)$} \\
\hline & $\boldsymbol{b}$ & se & $t$ & $d f$ & $p$ & fmi & b & se & $t$ & $d f$ & $p$ & fmi \\
\hline (Intercept) & -10.303 & 3.96 & -2.60 & 134.28 & .010 & .360 & -6.146 & 2.88 & -2.13 & 171.13 & .034 & .303 \\
\hline Well-being & 8.441 & 4.32 & 1.95 & 125.94 & .053 & .425 & 4.568 & 2.90 & 1.58 & 144.73 & .117 & .376 \\
\hline Male & NA & NA & NA & NA & NA & NA & -0.484 & 0.76 & -0.64 & 153.63 & .526 & .350 \\
\hline Age & -0.014 & 0.04 & -0.39 & 195.05 & .700 & .408 & 0.006 & 0.03 & 0.20 & 174.81 & .840 & .294 \\
\hline Education & 0.149 & 0.18 & 0.84 & 164.67 & .404 & .311 & 0.194 & 0.14 & 1.41 & 146.48 & .161 & .371 \\
\hline \multirow[t]{2}{*}{ PD symptoms } & 0.186 & 0.28 & 0.68 & 179.82 & .500 & .403 & -0.247 & 0.34 & -0.72 & 133.74 & .471 & .409 \\
\hline & \multicolumn{6}{|c|}{ Loose well-being criterion $\left(\right.$ Pseudo- $\left.\mathbf{R}^{2}=.117\right)$} & \multicolumn{6}{|c|}{ No GAD/PD/MDD symptoms $\left(\right.$ Pseudo-R $\left.^{2}=.226\right)$} \\
\hline Predictor & $\boldsymbol{b}$ & se & $t$ & $d f$ & $p$ & fmi & $\boldsymbol{b}$ & se & $t$ & $d f$ & $p$ & fmi \\
\hline (Intercept) & -2.342 & 1.58 & -1.48 & 143.77 & .141 & .378 & 0.730 & 1.38 & 0.53 & 147.29 & .598 & .368 \\
\hline Well-being & 1.715 & 1.60 & 1.07 & 126.65 & .286 & .431 & 0.140 & 1.40 & 0.10 & 124.05 & .920 & .440 \\
\hline Treatment & -0.008 & 0.02 & -0.44 & 127.87 & .660 & .427 & -0.037 & 0.03 & -1.37 & 76.01 & .174 & .627 \\
\hline GAD symptoms & -0.207 & 0.11 & -1.81 & 143.01 & .073 & .381 & -0.357 & 0.10 & -3.50 & 142.98 & .001 & .381 \\
\hline PD symptoms & -0.119 & 0.15 & -0.80 & 142.33 & .424 & .383 & -0.340 & 0.14 & -2.51 & 138.11 & .013 & .395 \\
\hline
\end{tabular}

Notes. $b=$ unstandardized regression coefficient; se $=$ standard error; $t=$ Wald $t$-ratio; $d f=$ small-sample multiple imputation degrees of freedom $p=$ two-sided $p$-value; fmi = fraction of missing information; Pseudo- $R^{2}=$ Nagelkerke $R^{2}$; Sex (i.e., Male) and GAD symptoms were removed from the regression equation for the strict well-being criterion because no male participants or participants with GAD symptoms at baseline met the criterion, causing their coefficients to be very unstable. 
Table 5. Mean differences of Well-being POMP total scores between Wave 1 and Wave 2.

\begin{tabular}{|c|c|c|c|c|c|c|c|c|c|c|c|c|c|c|}
\hline \multirow[b]{3}{*}{ OWB Operation } & \multicolumn{7}{|c|}{ No GAD/PD/MDD symptoms, but no well-being criteria } & \multicolumn{7}{|c|}{ Optimal Well-Being (OWB) } \\
\hline & \multirow[b]{2}{*}{$n$} & \multicolumn{2}{|c|}{ Wave 1} & \multicolumn{2}{|c|}{ Wave 2} & \multicolumn{2}{|c|}{ Change } & \multirow[b]{2}{*}{$n$} & \multicolumn{2}{|c|}{ Wave 1} & \multicolumn{2}{|c|}{ Wave 2} & \multicolumn{2}{|c|}{ Change } \\
\hline & & $M$ & $S D$ & $M$ & $S D$ & $\Delta M$ & $d$ & & $M$ & $S D$ & $M$ & $S D$ & $\Delta M$ & $d$ \\
\hline Strict & 50 & 0.65 & 0.12 & 0.72 & 0.11 & 0.07 & $.48^{*}$ & 6 & 0.81 & 0.13 & 0.92 & 0.04 & 0.11 & $1.02 *$ \\
\hline Medium & 46 & 0.65 & 0.12 & 0.71 & 0.10 & 0.06 & $.43^{*}$ & 10 & 0.76 & 0.15 & 0.88 & 0.06 & 0.12 & $1.01 *$ \\
\hline Loose & 22 & 0.63 & 0.10 & 0.62 & 0.09 & -0.01 & -0.07 & 34 & 0.69 & 0.14 & 0.81 & 0.07 & 0.12 & $1.00 *$ \\
\hline
\end{tabular}

Note. $\mathrm{d}=$ Cohen's d or the standardized mean difference; $\Delta \mathrm{M}=$ unstandardized mean difference; OWB Operation $=$ Well-being criteria used to define optimal well-being after psychopathology; $* \mathrm{p}<.05, * * \mathrm{p}<.01 ; * * * \mathrm{p}<.001$ 
Supplementary Materials for:

Optimal well-being in the aftermath of anxiety disorders:

\section{A 10-year longitudinal investigation}

This PDF file includes:

Page 2: A. Composite International Diagnostic Interview - Short Form (CIDI-SF)

Page 7: B. Well-being items and the Scales of Psychological Well-being

Page 12: C. Domain-specific Mean Differences

Page 12: D: Domain-specific Optimal Well-being (OWB)

Page 14: Tables S1, S2, and S3

Page 17: Supplementary References

Other supplementary files for this manuscript can be found on our OSF webpage: https://osf.io/puq8f/ in the "JAD Initial Submission" folder:

- $\quad$ midus longitudinal data probability sample 052917.sav = initial SPSS dataset

- HFAA_wellbeing_021520.R = R script for creating well-being variables

- HFAA_symptoms_021520 = R script for creating symptom variables

- HFAA_hfap_021520.R = R script for creating high functioning after psychopathology variables

- hfaa_data_full_030120.xlsx = Excel workbook dataset for analyses containing all cases

- hfaa_data_noDx_030120.xlsx = Excel workbook dataset for analyses containing cases without a disorder at Wave 1

- hfaa_data_yaDx_030120.xlsx = Excel workbook dataset for analyses containing cases with a disorder at Wave 1

- HFAA_analyses_021520.R = R script for conducting analyses 


\section{A. Composite International Diagnostic Interview - Short Form (CIDI-SF)}

\section{Generalized Anxiety Disorder:}

These questions were also administered during the phone interview and the scale was created using standard practice ${ }^{1,2}$. Specifically, those who were classified to have GAD if they said "more" to 1 , "most days" to "every day" for 2 , said "more than one" to item 3 or "yes" to item 4 and had a total score of 3 or more symptoms listed in items $5 \mathrm{a}-\mathrm{j}$. All others were classified to not have GAD.

1) People differ a lot in how much they worry. Considering how things have been going in your life over the PAST 12 MONTHS, do you worry MORE than most people in the same situation, LESS than most people, or ABOUT THE SAME as most people in the same situation? (responses: more, less, about the same, I don't worry at all)

2) Thinking about the PAST 12 MONTHS, did you worry... (READ LIST. responses: everyday, just about every day, most days, about half the days, or less than half the days)

3) Do you usually worry about ONE particular thing or MORE THAN ONE thing? (responses: one thing, more than one thing)

4) Do you ever have different worries on your mind AT THE SAME TIME?

5) Some people have physical reactions because of their worry. Thinking about the PAST 12 MONTHS, how often did you have each of the following reactions because of your worry? Include ONLY physical reactions that might have been caused by your worry, not those that were caused by something else. (First, how/How) often (over the PAST 12 MONTHS) (responses: most days, about half the days, less than half the days or never)
a. "were you restless because of your worry?"
b. "were you keyed up, on edge, or had a lot of nervous energy?"
c. "were you irritable because of your worry?"
d. "did you have trouble falling asleep?"
e. "did you have trouble staying asleep because of your worry?"
f. "did you have trouble keeping your mind on what you were doing?" 
g. "did you have trouble remembering things because of your worry?"

h. "were you low on energy?"

i. "did you tire easily because of your worry?"

j. "did you have sore or aching muscles because of tension?"

\section{Panic Disorder:}

These questions were administered during the phone interview and the scale was created using standard practice ${ }^{1,2}$. Specifically, those who were classified to have Panic Attacks if they said "yes" to item 1 or item 2, said "no" or "do not know" to item 3 and had three or more symptoms listed in items 4a-f. All others were classified to not have Panic Attacks.

1) DURING THE PAST 12 MONTHS, did you ever have a spell or an attack when ALL OF A SUDDEN you felt frightened, anxious, or very uneasy, in a situation when most people would not be afraid or anxious?

2) DURING THE PAST 12 MONTHS, did you ever have a spell or attack when for no reason your heart suddenly began to race, you felt faint, or you couldn't catch your breath? When we say, 'for no reason,' we mean that it was NOT due to any physical cause, like a heart problem.

3) Did (this attack happen in a situation/ALL of these attacks happen in situations) when you were in danger or were the center of attention?

4) Following associated symptoms:
a. "When you have attacks, does your heart pound?"
b. "(When you have attacks,) do you have tightness, pain, or discomfort in your chest or stomach?"
c. "(When you have attacks,) do you sweat?"
d. "(When you have attacks, ) do you tremble or shake?"
e. "(When you have attacks, ) do you have hot flashes or chills?"
f. "(When you have attacks, ) do you, or things around you, seem unreal?" 


\section{Major Depressive Disorder:}

These questions were administered during the phone interview and the scale was created using standard practice ${ }^{1,2}$. For detailed information on how this was administered see below. If they reported 4 or more symptoms listed under item 4 then they were considered to have depression. All other participants were not.

1) The next questions are about your mood. DURING THE PAST 12 MONTHS, was there ever a time when you felt sad, blue, or depressed for two weeks or more in a row? (responses: Yes; No; I did not feel depressed because I was on anti-depressant medication)

2) [IF Q1="YES"] Please think of THE TWO-WEEK PERIOD during the past 12 months when these feelings were worst. During that time, did the feelings of being sad, blue, or depressed usually last...(READ LIST) all day long; most of the day; about half the day; or less than half the day?

3) [IF Q2="ALL DAY LONG" OR "MOST OF THE DAY"] During the two weeks when these feelings were worst, how often did you feel this way... (READ LIST) every day; almost every day; or less often than that)?

4) [IF Q3=“EVERY DAY" OR "ALMOST EVERY DAY”] During two weeks in past 12 months, when you felt sad, blue, or depressed, did you... (responses: yes; no)

a. Lose of interest in most things?

b. Feel more tired out or low on energy than usual?

c. Lose your appetite?

i. [IF Q4C="NO"] Did your appetite INCREASE during those same two weeks?

d. Have more trouble falling asleep than you usually do during those two weeks?

i. [IF Q4D="YES"] Did that happen EVERY NIGHT, NEARLY EVERY NIGHT, or LESS OFTEN during those two weeks?

e. Have a lot more trouble concentrating than usual? 
f. People sometimes feel down on themselves, no good, or worthless. During that two week period, did you feel this way?

g. Did you think a lot about death -- either your own, someone else's, or death in general -- during those two weeks?

5) [IF (Q1=“NO" OR “DID NOT FEEL DEPRESSED BECAUSE...") OR (Q2=“ABOUT HALF THE DAY”, "LESS THAN HALF THE DAY”, OR "DON'T KNOW”) OR (Q3=“LESS OFTEN THAN THAT" OR “DON'T KNOW")] DURING THE PAST 12 MONTHS, was there ever a time lasting two weeks or more when you lost interest in most things like hobbies, work, or activities that usually give you pleasure? (responses: yes; no; I did not feel loss of interest because i was on anti-depressant medication)

6) [IF Q5=“YES”] Please think of THE TWO-WEEK PERIOD during the past 12 months when you had the MOST COMPLETE loss of interest in things. During that time, did the loss of interest usually last... (READ LIST) all day long; most of the day; about half the day; or less than half the day?

7) During the two weeks when these feelings were worst, how often did you feel this way...(READ LIST) every day; almost every day; or less often than that)?

8) Thinking about those same two weeks, did you...

a. Feel more tired out or low on energy than usual?

b. Lose your appetite?

i. [IF Q8B=“NO"] Did your appetite INCREASE during those same two weeks?

c. Have more trouble falling asleep than you usually do during those two weeks?

i. [IF Q8C="YES"] Did that happen EVERY NIGHT, NEARLY EVERY NIGHT, or LESS OFTEN during those two weeks?

d. Have a lot more trouble concentrating than usual?

e. People sometimes feel down on themselves, no good, or worthless. During that two week period, did you feel this way? 
f. Did you think a lot about death -- either your own, someone else's, or death in general -- during those two weeks? 


\section{B. Well-being items and the Scales of Psychological Well-being}

\section{Life Satisfaction Wave 1/Wave 2}

The life satisfaction question comes from the SAQ: "Using a scale from 0 to 10 where 0 means the 'worst possible life overall' and 10 means 'the best possible life overall', how would you rate your life overall these days?"
1) Overall life
2) Health
3) Work
4) Children
5) Marriage/Romantic Relationship

\section{Negative Affect Wave 1/Wave 2}

The following questions about positive affect were asked in the SAQ (response categories: "all of the time", "most of the time", "some of the time", "a little of the time", "none of the time"). During the past 30 days, how much of the time did you feel..."

1) So sad nothing could cheer you up

2) Nervous

3) Restless or fidgety

4) Hopeless

5) That everything was an effort

6) Worthless

\section{Positive Affect Wave 1/Wave2}

The following questions about positive affect were asked in the SAQ (response categories: "all of the time", "most of the time", "some of the time", "a little of the time", "none of the time").

During the past 30 days, how much of the time did you feel..."
1) Cheerful?
2) In good spirits? 

3) Extremely happy?
4) Calm and peaceful?
5) Satisfied?
6) Full of life?

\section{Psychological Well-Being (PWB) Wave 1}

Ryff's psychological well-being index, 3-item subscale version ${ }^{3}$, is based on 18 questions from the SAQ, with response categories: "agree strongly", "agree somewhat", "agree a little", "don't know", "disagree a little", "disagree somewhat", "disagree strongly". $\left({ }^{*}=\right.$ Reverse coded, items in dataset include A1SF1- with each corresponding letter)

\section{Self-Acceptance}

Please indicate how strongly you agree or disagree with each of the following statements:

1) I like most parts of my personality.

2) When I look at the story of my life, I am pleased with how things have turned out so far.

3) * In many ways I feel disappointed about my achievements in life.

\section{Purpose in Life}

1) Some people wander aimlessly through life, but I am not one of them.

2) *I live life one day at a time and don't really think about the future.

$3)$ *I sometimes feel as if I've done all there is to do in life.

\section{Environmental Mastery}
1) * The demands of everyday life often get me down.
2) In general, I feel I am in charge of the situation in which I live.
3) I am good at managing the responsibilities of daily life.

\section{Positive Relations with Others}

1) * Maintaining close relationships has been difficult and frustrating for me.

2) People would describe me as a giving person, willing to share my time with others.

$3)$ * I have not experienced many warm and trusting relationships with others. 


\section{Personal Growth}

1) For me, life has been a continuous process of learning, changing, and growth.

2) I think it is important to have new experiences that challenge how I think about myself and the world.

3) * I gave up trying to make big improvements or changes in my life a long time ago.

\section{Autonomy}

1) *I tend to be influenced by people with strong opinions.

2) I have confidence in my own opinions, even if they are different from the way most other people think.

3) I judge myself by what I think is important, not by the values of what others think is important.

\section{Psychological Well-Being (PWB) Wave 2}

Ryff's psychological well-being index, 7-item subscale version ${ }^{4}$, is based on 42 questions from the SAQ, with response categories: "agree strongly", "agree somewhat", "agree a little”, "don't know", "disagree a little", "disagree somewhat", "disagree strongly". ( ${ }^{*}=$ Reverse coded, items in dataset include BS1E1- with each corresponding letter)

\section{Autonomy}

1) I am not afraid to voice my opinions, even when they are in opposition to the opinions of most people

2) My decisions are not usually influenced by what everyone else is doing

$3)^{*}$ I tend to worry about what other people think of me

$4)^{*}$ I tend to be influenced by people with strong opinions

5) I have confidence in my opinions, even if they are contrary to general consensus

$6)$ * It is difficult for me to voice my opinions on controversial matters

7) I judge myself by what I think is important, not by the values of what others think is important

\section{Environmental Mastery}


1) In general, I feel I am in charge of the situation in which I live

2) * The demands of everyday life often get me down

$3)^{*}$ I do not fit very well with the people and the community around me

4) I am quite good at managing the many responsibilities of my daily life

5) * I often feel overwhelmed by my responsibilities

$6)^{*}$ I have difficulty arranging my life in a way that is satisfying to me

7) I have been able to build a home and a lifestyle for myself that is much to my liking

\section{Personal Growth}

1) * I am not interested in activities that will expand my horizons

2) I think it is important to have new experiences that challenge how you think about yourself and the world

3) * When I think about it, I haven't really improved much as a person over the years

4) I have the sense that I have developed a lot as a person over time

$5)^{*}$ I do not enjoy being in new situations that require me to change my old familiar ways of doing things

6) For me, life has been a continuous process of learning, changing, and growth

7) * I gave up trying to make big improvements or changes in my life a long time ago

\section{Positive Relations with Others}

1) Most people see me as loving and affectionate

2) * Maintaining close relationships has been difficult and frustrating for me

3) * I often feel lonely because I have few close friends with whom to share my concerns

4) I enjoy personal and mutual conversations with family members and friends

5) People would describe me as a giving person, willing to share my time with others

$6)$ * I have not experienced many warm and trusting relationships with others

7) I know that I can trust my friends, and they know they can trust me

\section{Purpose in Life}

1) *I live life one day at a time and don't really think about the future

2) I have a sense of direction and purpose in my life 
3) * My daily activities often seem trivial and unimportant to me

$4) *$ I don't have a good sense of what it is I'm trying to accomplish in life

5) I enjoy making plans for the future and working to make them a reality

6) Some people wander aimlessly through life, but I am not one of them

7) * I sometimes feel as if I've done all there is to do in life

\section{Self-acceptance}

1) When I look at the story of my life, I am pleased with how things have turned out

2) In general, I feel confident and positive about myself

3) * I feel like many of the people I know have gotten more out of life than I have

4) I like most aspects of my personality

5) * In many ways, I feel disappointed about my achievements in life

6) * My attitude about myself is probability not as positive as most people feel about themselves

7) When I compare myself to friends and acquaintances, it makes me feel good about who I am 


\section{Domain Specific Mean Differences}

We present descriptive data for the well-being dimensions. Table S2 presents the means and standard deviations of the 9 well-being scales separately by the clinical group and healthy group. As expected, the healthy group was higher on every well-being scale. The left columns present the standardized mean difference (i.e., Cohen's d) between the two groups. The largest differences were for the affect scales with the smallest differences for the autonomy, personal growth, and purpose in life scales.

\section{Domain Specific Optimal Well-being}

Domain-specific OWB at follow-up is presented in Table S3. Rates were similar across disorders, except for GAD which had lower rates for life satisfaction (GAD vs. PD: $\mathrm{d}=-4.1 \%, \mathrm{z}=-1.33, \mathrm{p}=.184 ;$ GAD vs. MDD: $\mathrm{d}=-7.7 \%, \mathrm{z}=-1.86, \mathrm{p}=.063)$ and personal growth (GAD vs. PD: $\mathrm{d}=-9.5 \%, \mathrm{z}=-1.85, \mathrm{p}=.064$; GAD vs. MDD: $\mathrm{d}=-7.0 \%$, $\mathrm{z}=-1.53, \mathrm{p}=.126$ ), although neither quite reached statistical significance. Participants in the healthy group compared to the clinical group had higher OWB rates for every wellbeing domain. The differences were most pronounced for life satisfaction $\left(\mathrm{d}=-6.9 \% ; \chi^{2}\right.$ $=9.61, \mathrm{p}=.001)$, positive affect $\left(\mathrm{d}=-7.1 \%, \chi^{2}=11.23, \mathrm{p}=.001\right)$, and positive relationships $\left(\mathrm{d}=-9.3 \%, \chi^{2}=12.85, \mathrm{p}<.001\right)$ where those in the healthy group had over double the rates of those in the clinical group.

We observed marginally significant differences in domain-specific OWB between the GAD and PD groups on the personal growth and life satisfaction dimensions. Rates of personal growth were low for the GAD group (4.8\%) compared with $11.8 \%$ and $14.3 \%$ for the MDD and PD groups, respectively. Compared with major depressive episodes and 
panic attacks, research suggests that GAD entails a more chronic, restrictive pattern of poor understanding/fear of emotions, intolerance of uncertainty, and avoidance, which may limit the curiosity and exploration that drives personal growth (Behar, Dimarco, Hekler, Mohlman, Staples, 2009). In contrast, individuals with a previous PD diagnosis showed the highest rates of personal growth, which were within $2 \%$ of the healthy group (16.0\%). Nobody in the GAD group reported elevated life satisfaction compared with $7.7 \%$ and $4.1 \%$ for the MDD and PD groups, respectively. We were particularly surprised by the life satisfaction difference since MDD is characterized by clinically low levels of positive affect and, when severe, the belief that life is not worth living. However, lower concurrent life satisfaction during a major depressive episode does not necessarily entail lower levels years in the future when the episode has gone away. 
Table S1. Well-being Score Reliability (Cronbach's Alpha)

\begin{tabular}{l|cc}
\hline \multicolumn{1}{c|}{ Scale } & Wave 1 & Wave 2 \\
\hline Life Satisfaction & .656 & .645 \\
Negative Affect & .866 & .854 \\
Positive Affect & .911 & .906 \\
Autonomy & .480 & .713 \\
Environmental Mastery & .520 & .777 \\
Personal Growth & .550 & .751 \\
Personal Relations with & .582 & .772 \\
Others & .365 & .708 \\
Purpose in Life & .587 & .837 \\
Self-Acceptance & .836 & .899 \\
Well-being total score &
\end{tabular}

Note. The SPWB subscales at Wave 1 contained 3 items while at Wave 2 they contained 7 items. 
Table S2. Descriptive statistics and group comparisons at Wave 1.

\begin{tabular}{l|cc|cc|cr}
\hline \multicolumn{1}{c|}{ Well-being Scale } & \multicolumn{2}{c|}{ Clinical Group } & \multicolumn{2}{c|}{ Healthy Group } & \multicolumn{2}{c}{ Cohen's d } \\
\hline Satisfaction with life & Mean & SD & Mean & SD & Estimate & $95 \%$ CI \\
Negative affect* & 7.01 & 1.47 & 7.98 & 1.09 & 0.83 & {$[0.68,0.98]$} \\
Positive affect & 3.84 & 0.83 & 4.59 & 0.45 & 1.40 & {$[1.22,1.58]$} \\
Autonomy & 2.75 & 0.77 & 3.53 & 0.62 & 1.18 & {$[1.01,1.35]$} \\
Environment mastery & 5.24 & 1.20 & 5.51 & 1.05 & 0.25 & {$[0.11,0.39]$} \\
Personal growth & 4.68 & 1.25 & 5.55 & 1.04 & 0.80 & {$[0.65,0.96]$} \\
Positive relationships & 5.81 & 1.20 & 6.07 & 0.95 & 0.26 & {$[0.12,0.40]$} \\
Purpose in life & 4.76 & 1.46 & 5.53 & 1.29 & 0.58 & {$[0.44,0.72]$} \\
Self-acceptance & 5.27 & 1.27 & 5.66 & 1.10 & 0.34 & {$[0.20,0.48]$} \\
\hline
\end{tabular}

Note. ${ }^{*}$ Reverse coded such that higher scores reflect greater well-being. 
Table S3. Domain-specific Optimal well-being (OWB) rates across disorders at Wave 2.

\begin{tabular}{|c|c|c|c|c|c|c|c|c|c|c|}
\hline \multirow[b]{2}{*}{ OWB OPERATION } & \multicolumn{2}{|c|}{ GAD } & \multicolumn{2}{|c|}{ PD } & \multicolumn{2}{|c|}{ MDD } & \multicolumn{2}{|c|}{ Clinical group } & \multicolumn{2}{|c|}{ Healthy group } \\
\hline & Count & Percent & Count & Percent & Count & Percent & Count & Percent & Count & Percent \\
\hline Data at wave 1 and 2 & 42 & $100.00 \%$ & 98 & $100.00 \%$ & 195 & $100.00 \%$ & 256 & $100.00 \%$ & 1207 & $100.00 \%$ \\
\hline Satisfaction with life & 0 & $0.0 \%^{\mathrm{a}}$ & 4 & $4.1 \%^{\mathrm{a}}$ & 15 & $7.7 \%^{\mathrm{a}}$ & 16 & $6.25 \%^{\mathrm{a}}$ & 159 & $13.2 \%^{\mathrm{b}}$ \\
\hline Negative affect* & 0 & $0.0 \%^{\mathrm{a}}$ & 0 & $0.0 \%^{\mathrm{a}}$ & 3 & $1.5 \%^{\mathrm{a}}$ & 3 & $1.2 \%^{\mathrm{a}}$ & 39 & $3.2 \%{ }^{\mathrm{a}}$ \\
\hline Positive affect & 2 & $4.8 \%{ }^{\mathrm{a}}$ & 4 & $4.1 \%^{\mathrm{a}}$ & 11 & $5.6 \%^{\mathrm{a}}$ & 12 & $4.7 \% \%^{\mathrm{a}}$ & 142 & $11.8 \%^{\mathrm{b}}$ \\
\hline Autonomy & 3 & $7.1 \%^{\mathrm{a}}$ & 9 & $9.2 \%^{\mathrm{a}}$ & 21 & $10.8 \%{ }^{\mathrm{a}}$ & 26 & $10.2 \%^{\mathrm{a}}$ & 202 & $16.7 \%^{\mathrm{b}}$ \\
\hline Environment mastery & 4 & $9.5 \%{ }^{\mathrm{a}}$ & 9 & $9.2 \%{ }^{\mathrm{a}}$ & 21 & $10.8 \%^{\mathrm{a}}$ & 28 & $10.9 \%^{\mathrm{a}}$ & 213 & $17.6 \%^{\mathrm{b}}$ \\
\hline Personal growth & 2 & $4.8 \%^{\mathrm{a}}$ & 14 & $14.3 \%^{\mathrm{a}}$ & 23 & $11.8 \%{ }^{\mathrm{a}}$ & 33 & $12.9 \%^{\mathrm{a}}$ & 193 & $16.0 \%^{\mathrm{a}}$ \\
\hline Positive relationships & 5 & $11.9 \%{ }^{\mathrm{a}}$ & 9 & $9.2 \%{ }^{\mathrm{a}}$ & 20 & $10.3 \%^{\mathrm{a}}$ & 25 & $9.8 \%^{\mathrm{a}}$ & 231 & $19.1 \%^{\mathrm{b}}$ \\
\hline Purpose in life & 3 & $7.1 \%^{\mathrm{a}}$ & 9 & $9.2 \%{ }^{\mathrm{a}}$ & 16 & $8.2 \%^{\mathrm{a}}$ & 24 & $9.4 \%{ }^{\mathrm{a}}$ & 205 & $17.0 \%^{\mathrm{b}}$ \\
\hline Self-acceptance & 3 & $7.1 \%^{\mathrm{a}}$ & 12 & $12.2 \%^{\mathrm{a}}$ & 19 & $9.7 \%^{\mathrm{a}}$ & 26 & $10.2 \%^{\mathrm{a}}$ & 232 & $19.2 \%^{\mathrm{b}}$ \\
\hline
\end{tabular}

Note. $\mathrm{GAD}=$ generalized anxiety disorder; $\mathrm{PD}=$ panic disorder; $\mathrm{MDD}=$ major depressive disorder; Any Disorder $=\mathrm{GAD}$ and/or PD and/or MDD; No Disorder = No GAD and no PD and no MDD; *Reverse coded such that higher scores reflect greater well-being; a,b differently lettered superscripts indicate significantly different proportions $(\mathrm{p}<.05)$ and are separate for 1) GAD vs. PD vs. MDD and 2) Clinical group vs. Healthy group. 


\section{SI References}

1. Kessler, RC, Andrews G, Mroczek D, Ustun TB, Wittchen H- (1998) The World Health Organization Composite International Diagnostic Interview Short Form (CIDI-SF). International Journal of Methods in Psychiatry Research, 7(4), 171185.

2. Behar, E., Dimarco, I. D., Hekler, E. B., Mohlman, J., \& Staples, A. M. (2009). Current theoretical models of generalized anxiety disorder (GAD): Conceptual review and treatment implications. Journal of Anxiety Disorders, 23(8), 10111023.

3. Nelson CB, Kessler RC, Mroczek D (2001) Scoring the World Health Organization's Composite International Diagnostic Interview Short Form (CIDSF; v1.0 NOV98)

4. Ryff, C. D., \& Keyes, C. L. M. (1995). The structure of psychological well-being revisited. Journal of Personality and Social Psychology, 69(4), 719-729.

5. Van Dierendonck, D. (2004). The construct validity of Ryff's Scales of Psychological Well-being and its extension with spiritual well-being. Personality and Individual Differences, 36(3), 629-643. 